\title{
Mesoporous Graphene Hosts for Dendrite-Free Lithium Metal Anode in Working Rechargeable Batteries
}

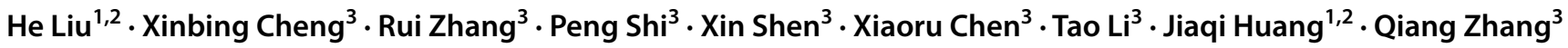

Received: 23 January 2020 / Revised: 2 February 2020 / Accepted: 3 February 2020 / Published online: 6 March 2020

(c) The Author(s) 2020

\begin{abstract}
Lithium (Li) metal anode has received extensive attentions due to its ultrahigh theoretical capacity and the most negative electrode potential. However, dendrite growth severely impedes the practical applications of the Li metal anode in rechargeable batteries. In this contribution, a mesoporous graphene with a high specific surface area was synthesized to host the Li metal anode. The mesoporous graphene host (MGH) has a high specific surface area $\left(2090 \mathrm{~m}^{2} / \mathrm{g}\right)$, which affords free space and an interconnected conductive pathway for Li plating and stripping, thus alleviating the volume variation and reducing the generation of dead Li during repeated cycles. More importantly, the high specific surface area of MGH efficiently reduces the local current density of the electrode, which favors a uniform Li nucleation and plating behavior, rendering a dendritefree deposition morphology at a low overpotential. These factors synergistically boost the Li utilization $(90.1 \%$ vs. $70.1 \%$ for $\mathrm{Cu}$ foil) and life span ( 150 cycles vs. 100 cycles for $\mathrm{Cu}$ foil) with a low polarization of MGH electrode at an ultrahigh current of $15.0 \mathrm{~mA} / \mathrm{cm}^{2}$. The as-prepared MGH can provide fresh insights into the electrode design of the Li metal anode operating at high rates.
\end{abstract}

Keywords Lithium metal anode $\cdot$ Mesoporous graphene hosts $\cdot$ Dendrite-free plating behavior $\cdot$ Working rechargeable batteries $\cdot$ Composite electrode

\section{Introduction}

Lithium (Li) ion batteries (LIBs) have realized commercial applications in portable devices, including smart phones, computers, and electric vehicles [1]. Compared with conventional fuel vehicles, electric vehicles are ready to innovate nearly every aspect of transportation, such as potentially reducing costs and carbon emissions and even changing driving habits [2]. A question pertaining to electric vehicles

Electronic supplementary material The online version of this article (https://doi.org/10.1007/s12209-020-00241-z) contains supplementary material, which is available to authorized users.

Qiang Zhang

zhang-qiang@mails.tsinghua.edu.cn

1 School of Materials Science and Engineering, Beijing Institute of Technology, Beijing 100081, China

2 Advanced Research Institute of Multidisciplinary Science, Beijing Institute of Technology, Beijing 100081, China

3 Beijing Key Laboratory of Green Chemical Reaction Engineering and Technology, Department of Chemical Engineering, Tsinghua University, Beijing 100084, China is how far they can go, which is mostly determined by the cell systems. Therefore, a high energy density battery is the primary demand for a long-range electric vehicle [3]. Compared with the graphite anode in commercial LIBs, the Li metal anode has a high theoretical capacity of $3860 \mathrm{mAh} / \mathrm{g}$ ( $372 \mathrm{mAh} / \mathrm{g}$ for the graphite anode) and the most negative potential of $-3.04 \mathrm{~V}$ vs. SHE (usually $0.1 \mathrm{~V} \mathrm{vs.} \mathrm{Li}^{+} / \mathrm{Li}$ for the graphite anode) [4,5]. Li metal batteries (LMBs) exhibit a high energy density and have the potential for practical applications in the top-end electronics, unmanned aerial vehicles, and electric vehicles [6-9].

The substitution of graphite anodes in commercial LIBs by Li metal anodes leads to a paradigm shift in the energy storage mechanism. In LIBs, Li ions can be intercalated into/ deintercalated from the intercalated graphite compounds during repeated charge/discharge processes. By contrast, the "hostless" nature of conversion-type Li metal anode indicates that the electrode suffers from infinite relative volume change during each cycle $[10,11]$. This "hostless" plating process induces considerable internal stress fluctuation of electrodes and deteriorates battery performance [12]. Another key issue of LMBs is the uncontrollable Li dendrite growth caused by 
the ultrahigh reactivity of Li metal, unstable solid-electrolyte interphase (SEI), and inhomogeneous current and $\mathrm{Li}$-ion flux distribution on the anode surface $[13,14]$. These issues collectively contribute to the short life span, reduced Coulombic efficiency (CE), low energy density, and poor safety performance of LMBs $[15,16]$.

Strategies are proposed to alleviate dendrite growth of the Li metal anode, such as constructing a stable SEI layer on the surface of Li metal electrode by regulating the components of electrolytes/additives and ex situ techniques [17-27], and utilizing solid-state electrolytes with high mechanical modulus [28-32]. The large volume variation in the "hostless" $\mathrm{Li}$ metal anode can only be remitted by providing a host framework to accommodate the deposited Li metal. Currently, 3D conductive metal/alloy current collectors $[33,34]$ and porous carbon-based frameworks [35, 36], as well as nonconductive 3D porous hosts [37], are proposed. Among them, porous carbon materials are regarded as superior hosts for Li deposition due to their high electronic conductivity, lightweight, superior electrochemical stability, high mechanical flexibility, and low cost [38]. Aside from providing space for Li deposition, 3D conductive host with a high specific surface area can also reduce the local current density of the electrode and delay the onset time of dendrite formation according to the classical Sand's time model $[39,40]$. Modification on the surface chemistry of carbon hosts can also regulate the nucleation and plating behaviors of Li metal, thus achieving a dendrite-free $\mathrm{Li}$ deposition morphology [41, 42]. Therefore, the use of efficient carbon hosts is highly expected to simultaneously address the large volume change and dendrite growth issues of working Li metal anodes.

In this contribution, a mesoporous graphene with a high specific surface area was prepared to host the Li metal anode. The mesoporous graphene host (MGH) can provide free space and an interconnected conductive pathway for Li plating and stripping, therefore alleviating the volume variation and reducing the generation of dead $\mathrm{Li}$ during repeated cycles. In addition, the higher specific surface area of MGH than that of flat $\mathrm{Li}$ foil anode efficiently reduces the local current density of the electrode, which favors a uniform Li nucleation and plating behavior, rendering a dendrite-free deposition morphology. These factors synergistically boost Li utilization, safety performance, and life spans of the Li metal anode at an ultrahigh current of $15.0 \mathrm{~mA} / \mathrm{cm}^{2}$. Therefore, the as-prepared MGH helps achieve a high-rate electrochemical performance of the Li metal anode, which is promising in practical applications.

\section{Experimental Section}

\section{Synthesis of Mesoporous Graphene}

The mesoporous graphene with an ultrahigh specific surface area was obtained via the chemical vapor deposition method by direct carbonization of $\mathrm{CH}_{4}$ and $\mathrm{MgO}$ template [43]. The $\mathrm{MgO}$ template was obtained via the hydrothermal reaction, with commercial $\mathrm{MgO}$ powders as raw materials. The tube containing the as-obtained mesoporous $\mathrm{MgO}$ template was heated to $950{ }^{\circ} \mathrm{C}$ in a tube furnace at a heating rate of $10^{\circ} \mathrm{C} /$ min with the protection of flowing $\mathrm{Ar}$ atmosphere. Then, the mixture of $\mathrm{CH}_{4}$ and $\mathrm{H}_{2}$ was simultaneously introduced into the fluidized bed reactor. $\mathrm{CH}_{4}$ was decomposed into carbon and deposited onto the $\mathrm{MgO}$ template. Subsequently, the furnace was naturally cooled to room temperature with the protection of $\mathrm{Ar}$ and $\mathrm{H}_{2}$. The as-prepared products were purified by $6.0 \mathrm{~mol} / \mathrm{L}$ hydrochloric acid at $80^{\circ} \mathrm{C}$ for $24.0 \mathrm{~h}$ to remove the $\mathrm{MgO}$ templates. Eventually, the mesoporous graphene was collected after washing, filtering, and freeze-drying.

\section{Characterizations}

The morphology and microstructures were investigated using a scanning electron microscope (SEM, JEOL JSM$7401 \mathrm{~F}$, Japan) operated at $3.0 \mathrm{kV}$ and a transmission electron microscope (TEM, JEOL-2010, Japan) with an accelerating voltage of $120 \mathrm{kV} . \mathrm{N}_{2}$ isotherms were obtained with an Autosorb-IQ ${ }_{2}$-MPC system (Quantachrome, USA). The specific surface area was calculated by the multipoint Brunauer-Emmett-Teller (BET) method.

\section{Electrochemical Measurements}

Standard CR2025 coin cells with a Celgard 2400 separator were assembled in an argon-filled glove box with $\mathrm{H}_{2} \mathrm{O}$ and $\mathrm{O}_{2}$ contents both below $10^{-6}$. All electrochemical measurements were conducted on a Neware multichannel battery cycler. For the half-cell test, the slurry of MGH powder and polyvinylidene fluoride binder (mass ratio of 85:15) in $\mathrm{N}$-methyl-2-pyrrolidone was coated on a $\mathrm{Cu}$ foil and dried in vacuum. The as-obtained $\mathrm{MGH}$-coated $\mathrm{Cu}$ foils were punched into $\Phi 13 \mathrm{~mm}$ disks as the working electrodes. $\mathrm{Li}$ metal foils with a thickness of $0.5 \mathrm{~mm}$ and a diameter of $16.0 \mathrm{~mm}$ (China Energy Lithium Co., Ltd.) were utilized as the counterelectrodes. $\mathrm{Cu}$ foils were used as the working electrodes in the control samples. The assembled LillCu halfcells were used to investigate the nucleation overpotential, plating/stripping behavior, and CEs of Li metal electrode with the electrolyte of $3.0 \mathrm{~mol} / \mathrm{L}$ lithium bis(fluorosulfonyl) imide in 1,2-dimethoxyethane. The dosage of the electrolyte 
in each cell was $60 \mu \mathrm{L}$. The concentrated electrolyte was selected to construct a stable SEI, excluding the effect of SEI instability on the function of MGH. In the long-term stability test, a pre-cycle at $0.5 \mathrm{~mA} / \mathrm{cm}^{2}$ was conducted to construct a stable SEI on the surface of the electrode. The impedance measurements were conducted on a Solartron 1470 E electrochemical workstation (Solartron Analytical) over the frequency ranging from $100 \mathrm{kHz}$ to $0.01 \mathrm{~Hz}$.

\section{Results and Discussion}

\section{Characterization of Mesoporous Graphene}

The as-obtained mesoporous graphene exhibits a porous and hexagonal morphology (Fig. 1a, b). The lateral size and thickness of graphene layers are approximately $2 \mu \mathrm{m}$ and $50 \mathrm{~nm}$, respectively (Fig. S1). The few-layered graphene flakes wrinkle into a honeycombed framework with in-plane nanocages in the size of 5-10 nm (Fig. 1c). Numerous defective sites are generated at the corners or edges of these nanocages, which favor the absorption of $\mathrm{Li}$ ions, and correspond to the nanocages in the TEM image. The BET surface area of 3D porous graphene reaches as high as $2090 \mathrm{~m}^{2} / \mathrm{g}$ (Fig. 1d), which is expected to considerably reduce the local current density of the Li metal electrode and suppress dendrite growth at a high current rate.
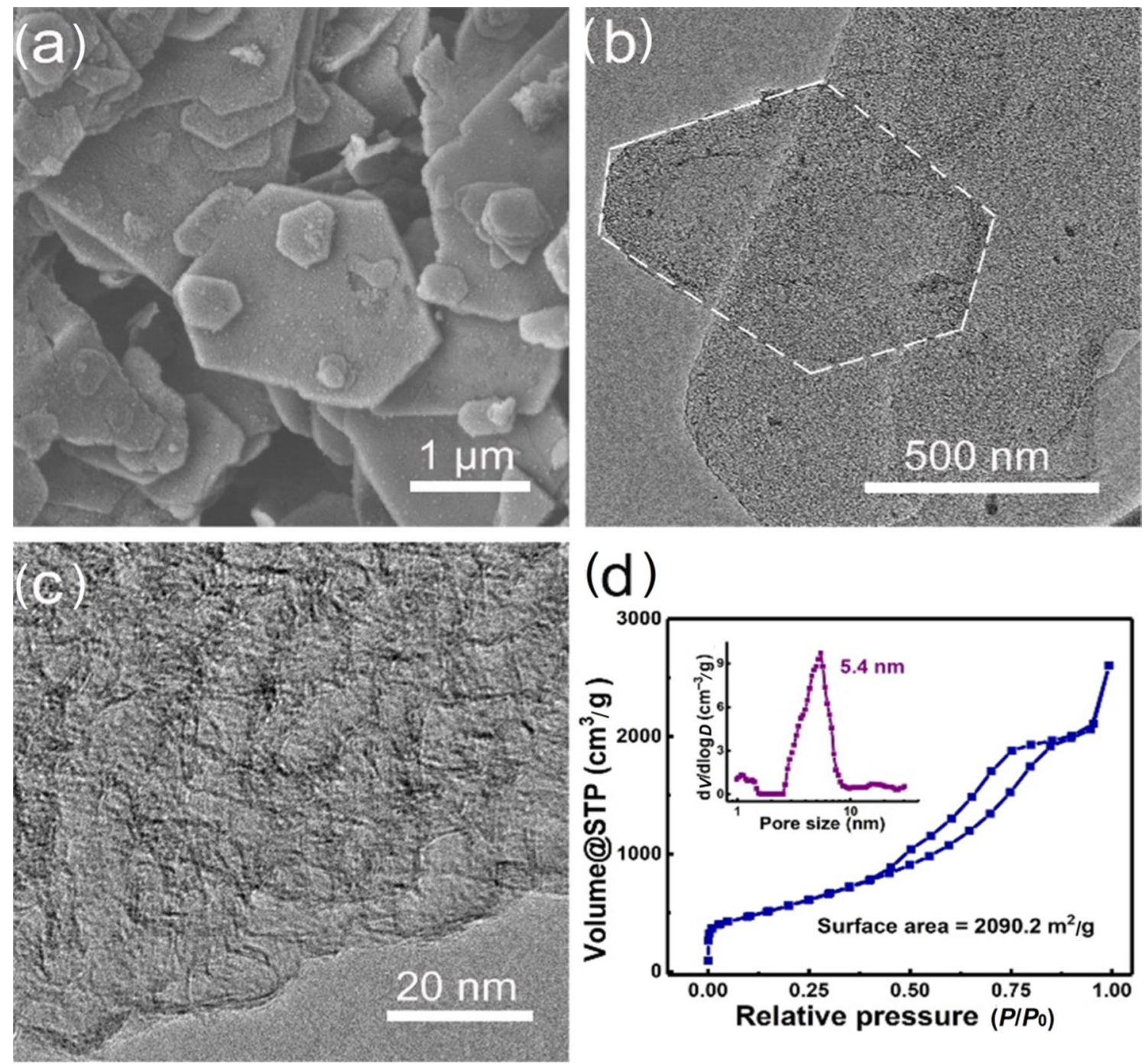

Fig. 1 Characterizations of mesoporous graphene: a SEM image, b low-resolution and $\mathbf{c}$ high-resolution TEM images of MGH materials, $\mathbf{d} \mathrm{N}_{2}$ sorption isotherms of MGH materials and corresponding pore size distribution 


\section{Li Nucleation and Deposition Behavior on MGH Electrode}

The Li nucleation overpotential on MGH and $\mathrm{Cu}$ foil electrodes at different current densities is compared to evaluate the Li nucleation and growth behavior. The overpotentials between these two electrodes during electrochemical deposition are the nucleation and masstransfer overpotentials. The nucleation overpotential is mainly influenced by the surface chemistry of the electrode, whereas the masstransfer overpotential depends on the applied current density and Li-ion migration properties. The stable platform voltage in the voltage-time profiles represents the masstransfer overpotential, and the value increases with large current densities (Fig. 2a, b). The nucleation overpotential is applied to evaluate the nucleation resistance and defined by the difference between masstransfer overpotential and sharp tip voltage [44]. Obviously, the Li nucleation overpotentials on MGH electrode are lower than those on $\mathrm{Cu}$ foil (Fig. 2). High nucleation overpotentials of $20-44 \mathrm{mV}$ are observed on $\mathrm{Cu}$ foil even at low current rates ranging from $0.01 \mathrm{~mA} / \mathrm{cm}^{2}$ to $0.1 \mathrm{~mA} / \mathrm{cm}^{2}$. By contrast, a low nucleation overpotential of $10 \mathrm{mV}$ is observed on MGH electrode at $0.1 \mathrm{~mA} / \mathrm{cm}^{2}$. The nucleation difference between these two electrodes can be attributed to their surface properties [45-48]. The ultrahigh specific surface area of MGH $\left(2090 \mathrm{~m}^{2} / \mathrm{g}\right)$ compared with that of flat $\mathrm{Cu}$ foil effectively decreases the local current density of the electrode at the same bulk current density. The numerous defective sites generated at the corners or edges of graphene nanocages also favor the absorption and nucleation of $\mathrm{Li}$ ions, thus reducing the nucleation resistance of metallic Li. The Li nucleation overpotential on MGH is only $17 \mathrm{mV}$ even at a current of $1.0 \mathrm{~mA} / \mathrm{cm}^{2}$; however, this value increases to $79 \mathrm{mV}$ on $\mathrm{Cu}$ foil. This finding further indicates that MGH with a high specific surface area can efficiently regulate the nucleation behavior of the Li metal anode at high current densities.

SEM images are taken to compare the Li nucleation and deposition behavior on MGH and $\mathrm{Cu}$ foil electrodes visually. Striking differences in Li plating morphology can be observed between these two electrodes (Figs. 3 and S2). Dendritic $\mathrm{Li}$ deposition is observed on $\mathrm{Cu}$ foil even at a low capacity of $0.1 \mathrm{mAh} / \mathrm{cm}^{2}$, and the deposits are distributed on $\mathrm{Cu}$ foil randomly (Figs. 3a and S2a). This nonuniform deposition behavior is induced by the inhomogeneous local current density on rough $\mathrm{Cu}$ foil surface. The nonuniform surface chemistry of $\mathrm{Cu}$ foil also leads to the inhomogeneity in Li-ion concentration distribution, resulting in the uncontrollable dendritic $\mathrm{Li}$ deposition on $\mathrm{Cu}$ foil electrode. The dendrites grow longer and thicker with the increase in capacity (Fig. 3b, c). The diameter of these dendrites ranges from several to a dozen micrometers at $2.0 \mathrm{mAh} / \mathrm{cm}^{2}$, which leads to poor efficiency and high safety risk of LMBs.

By contrast, a uniform Li deposition behavior without any dendrite can be observed on MGH electrode (Fig. S2d-f). A porous framework is constructed by randomly stacking hexagonal graphene layers in MGH before Li plating (Fig. $\mathrm{S} 2 \mathrm{~d})$. The deposited Li fills in the interspace between graphene layers at $0.5 \mathrm{mAh} / \mathrm{cm}^{2}$ (Fig. S2e). Notably, a thin $\mathrm{Li}$ layer covers the MGH electrode uniformly with the increase in plating capacity to $2.0 \mathrm{mAh} / \mathrm{cm}^{2}$, rather than forming $\mathrm{Li}$ dendrites (Figs. $3 \mathrm{f}$ and S2f). Further observation of morphological evolution under high magnification clearly depicts the deposition process (Fig. 3d-f). Li particles with dozens of nanometers in size uniformly distribute on the MGH surface at a low plating capacity of $0.1 \mathrm{mAh} / \mathrm{cm}^{2}$ (Fig. 3d). The MGH is wrapped by more densely and uniformly distributed Li nuclei as the capacity is increased to $0.5 \mathrm{mAh} / \mathrm{cm}^{2}$ (Fig. 3e). The uniform nucleation sites in MGH electrodes further contribute to the uniform deposition behavior, leading to the propagation and coalescence of these particles
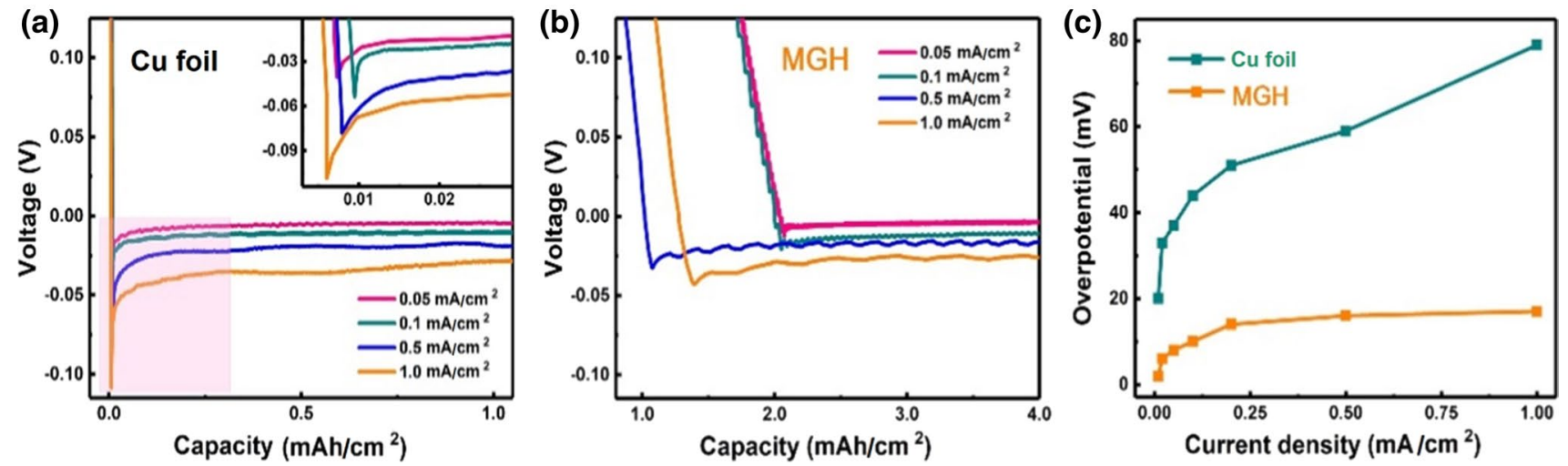

Fig. 2 Comparison of the nucleation overpotentials between $\mathrm{Cu}$ foil and MGH electrodes. The voltage-time curves during Li nucleation at different current densities on $\mathbf{a} \mathrm{Cu}$ foil and $\mathbf{b}$ MGH electrodes. $\mathbf{c}$
The $\mathrm{Li}$ nucleation overpotentials on $\mathrm{Cu}$ foil surface and MGH electrodes at different current densities 

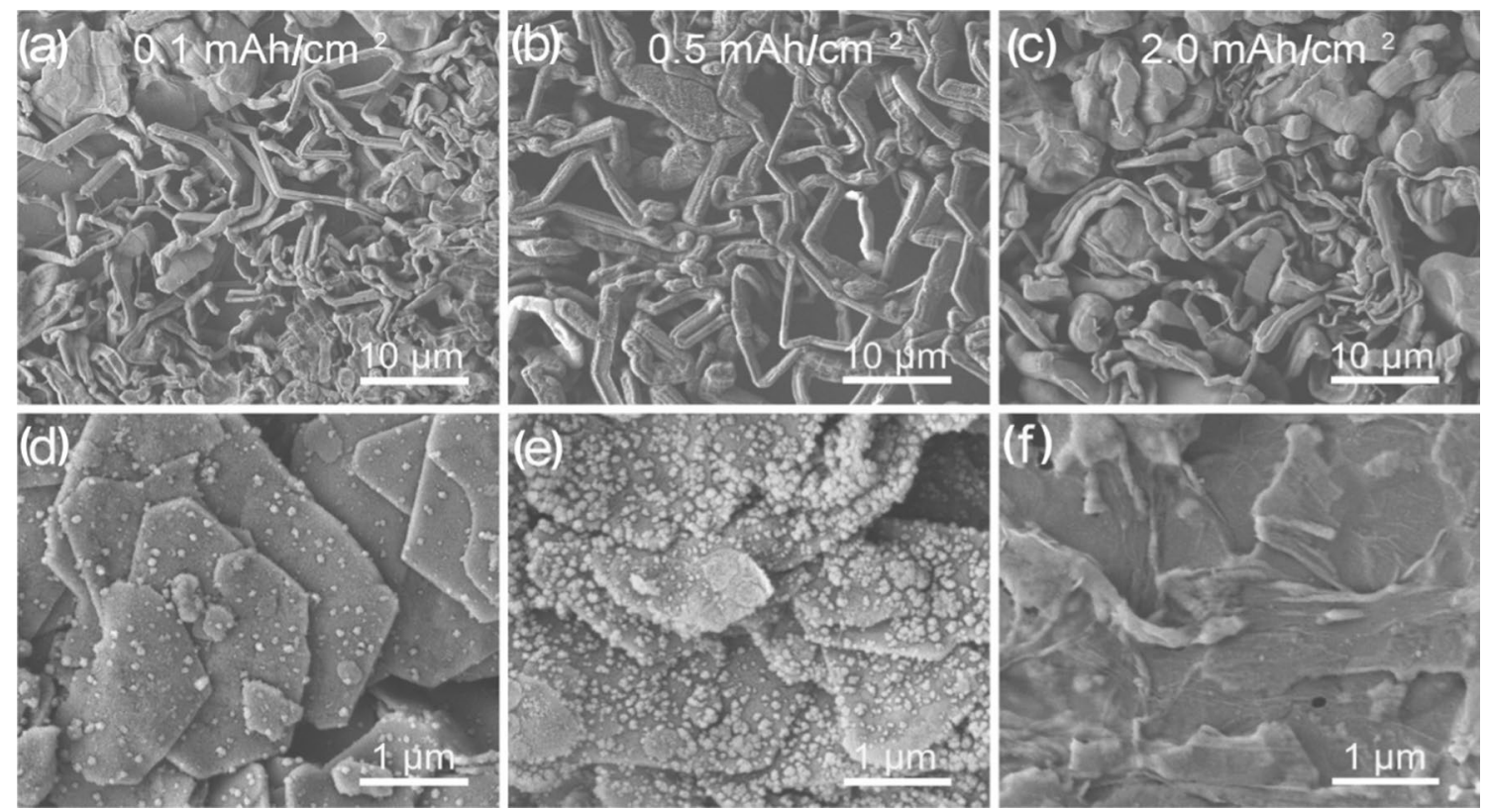

Fig. 3 Li deposition morphology on MGH and Cu foil electrodes, SEM images of Li deposits on a-c Cu foil and d-f MGH electrodes after plating different capacities of $\mathrm{Li}$ at a current of $0.5 \mathrm{~mA} / \mathrm{cm}^{2}$

with the increasing lithiation capacity. Finally, a smooth Li depositing layer covers the MGH surface and fills the gaps (Fig. 3f). The contrast of Li deposition morphological evolution between $\mathrm{MGH}$ and $\mathrm{Cu}$ foil electrodes underscores the significance of the high specific surface area of MGH materials in reducing the local current density and regulating the nucleation and deposition behavior of $\mathrm{Li}$ ions.

\section{Long-Term Stability of the MGH Electrode}

The $\mathrm{CE}$ and long-term stability of the MGH and $\mathrm{Cu}$ foil electrodes were investigated. Obviously, the MGH electrode exhibits a higher $\mathrm{CE}$ and longer life span than the $\mathrm{Cu}$ foil electrode at different current densities at $2.0 \mathrm{mAh} / \mathrm{cm}^{2}$ (Fig. 4a). At rates of 2.0 and $5.0 \mathrm{~mA} / \mathrm{cm}^{2}$, the MGH electrode exhibits high CEs of $98.9 \%$ and $98.4 \%$ for 340 and 280 cycles, respectively, indicating highly reversible $\mathrm{Li}$ in the MGH electrode. By contrast, the life span of the $\mathrm{Cu}$ foil electrode under the same conditions is half that of the MGH electrode with lower CEs (97.0\% and $96.0 \%$ at rates of 2.0 and $5.0 \mathrm{~mA} / \mathrm{cm}^{2}$, respectively). Notably, high CEs of $97.7 \%$ and $90.1 \%$ can be retained in the MGH electrode at ultrahigh rates of 10.0 and $15.0 \mathrm{~mA} / \mathrm{cm}^{2}$, respectively, for approximately 150 cycles. However, the $\mathrm{Cu}$ foil electrode exhibits a poor electrochemical performance with fluctuant CEs and sharply reduced life span at such high currents. These results can be attributed to the uncontrollable dendrite growth and fragile SEI layers on the $\mathrm{Cu}$ foil electrode. The higher CE and longer service time of the MGH electrode than the $\mathrm{Cu}$ foil electrode validate the superiority of the high specific surface area in decreasing local current density and boosting Li utilization, particularly at high currents.

The MGH electrode exhibits low polarization during the entire cycling process (Fig. 4b, c). In Cu foil electrode, polarization increases from 150 to $230 \mathrm{mV}$ after 150 cycles. This process is accompanied by the pulverization and volume expansion of the Li metal anode, as well as the accumulation of dead $\mathrm{Li}$, leading to the increase in $\mathrm{Li}$-ion transfer resistance. In comparison, the hysteresis of the MGH electrode increases from 100 to $150 \mathrm{mV}$ during the initial 70 cycles to form a stable SEI layer on the Li metal electrode. Afterward, a stable plateau is maintained for 180 cycles, indicating the excellent long-term stability of the MGH electrode. In addition, the hysteresis of the MGH electrode during the fifth cycle is relatively lower than that of the $\mathrm{Cu}$ foil electrode at different rates (Fig. S3). In particular, a $50 \mathrm{mV}$ lower hysteresis can be observed in the MGH electrode than in the $\mathrm{Cu}$ foil electrode at $15.0 \mathrm{~mA} / \mathrm{cm}^{2}$. On the one hand, MGH can afford a free space for Li deposition and alleviate the volume variation during cycling. On the other hand, the conductive framework of MGH provides an interconnected pathway for electron transfer, alleviating the formation of dead Li. The considerably decreased local current density by the high specific surface area [40] of the MGH electrode also contributes to the stable cycling behavior.

Electrochemical impedance spectroscopy was conducted to investigate the Li-ion diffusion behavior in different electrodes. The MGH electrode exhibits a lower and more stable SEI resistance (semicircle at mid-frequency) than the $\mathrm{Cu}$ foil electrode (Fig. S4), indicating that a stable and ionically 


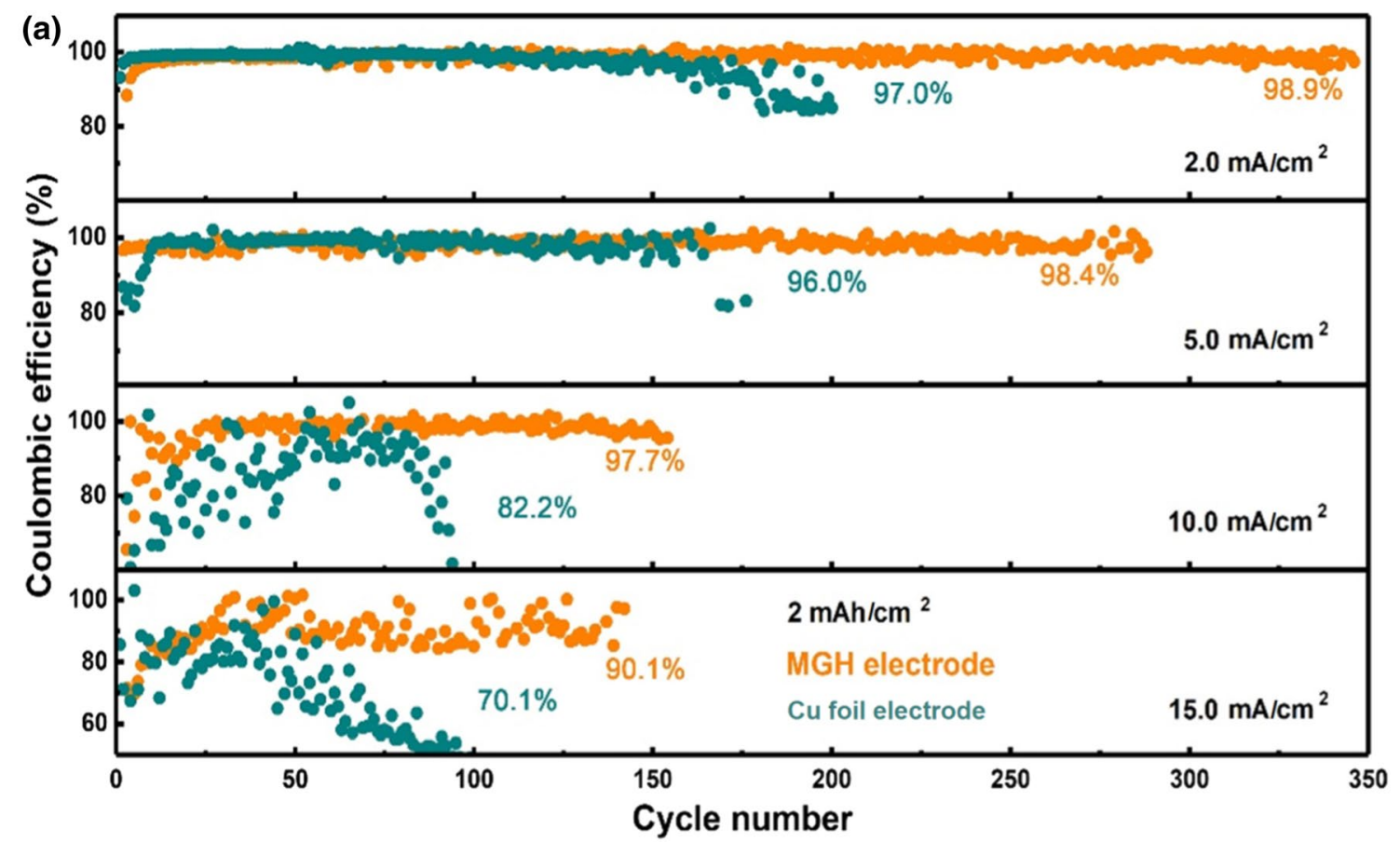

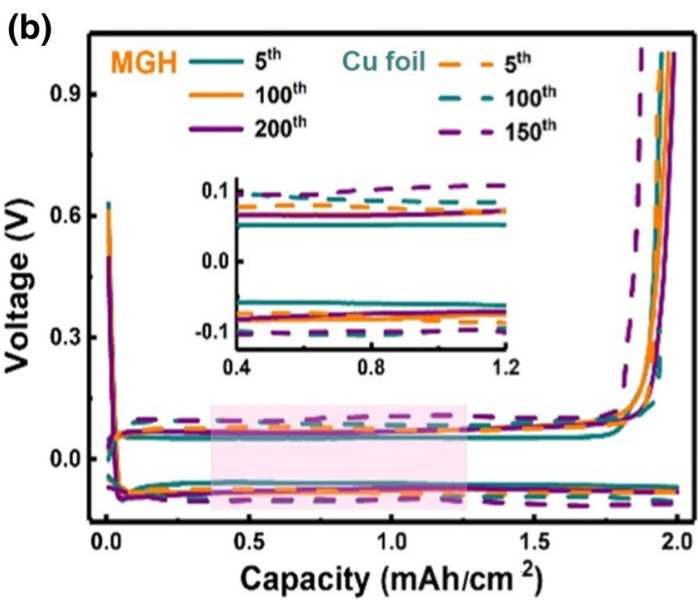

Fig. 4 Long-term cycles of different electrodes, a CEs of MGH and $\mathrm{Cu}$ foil electrodes at different rates with a capacity of $2.0 \mathrm{mAh} / \mathrm{cm}^{2}$, b voltage profiles of $\mathrm{MGH}$ and $\mathrm{Cu}$ foil electrodes at $5.0 \mathrm{~mA} / \mathrm{cm}^{2}$, c

conductive SEI layer is constructed between the interfaces. These advantages render a stable and low-voltage plateau in the MGH electrode upon stabilization of the SEI layer.

The morphologies of the MGH and $\mathrm{Cu}$ foil electrodes after long cycles at different current densities were investigated. For the MGH electrode, the deposited Li metal uniformly covers the hexagonal graphene layers and dendrites do not grow in their gaps even after 200 cycles at high rates of 2.0 and $5.0 \mathrm{~mA} / \mathrm{cm}^{2}$ (Fig. S5a, b). The uniform deposition behavior can be retained for 100 cycles at ultrahigh currents of 10.0 and $15.0 \mathrm{~mA} / \mathrm{cm}^{2}$ (Fig. S5c, d). The conductive MGH with high specific surface area also favors a uniform

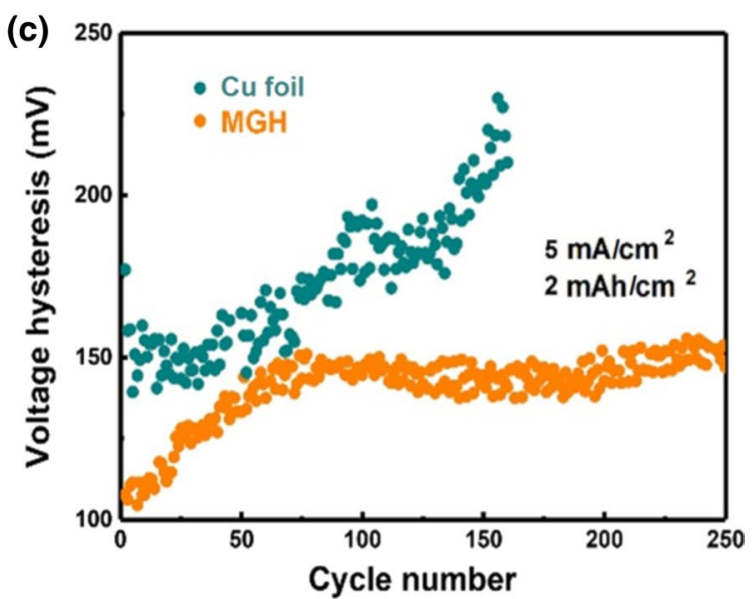

comparison of the polarization voltage between $\mathrm{MGH}$ and $\mathrm{Cu}$ foil electrodes at $5.0 \mathrm{~mA} / \mathrm{cm}^{2}$

stripping behavior during long cycles, mitigating the formation of dead $\mathrm{Li}$ and facilitating the generation of superior SEI film (Fig. S6a-d). While for the $\mathrm{Cu}$ foil electrode, random and loose dendrites are observed during long cycles even at $2.0 \mathrm{~mA} / \mathrm{cm}^{2}$ (Fig. S5e-h). These loose structures are easily detached from the conductive $\mathrm{Cu}$ foil substrate during the stripping process, leading to the formation of dead $\mathrm{Li}$ (Fig. S6e-h). The accumulation of dead $\mathrm{Li}$ and ruptured SEI layers leads to the increase in ionic transfer resistance (Fig. S4) and polarization voltage (Fig. 4c). These results indicate that MGH can not only regulate the $\mathrm{Li}$ nucleation and deposition behavior at the initial stage but also maintain 
the stability of the framework and work well during long repeated cycles.

\section{Conclusions}

Conductive MGH materials with a high specific surface area were designed to host the Li metal anode with a high-rate electrochemical performance. The following advantages can be provided by this unique host framework:

(1) The high specific surface area of MGH can efficiently reduce the local current density of the electrode, rendering uniform nucleation, and deposition of $\mathrm{Li}$ ions without any dendrites.

(2) MGH provides an interconnected conductive pathway for Li plating and stripping, thus alleviating the formation of dead Li.

(3) MGH can maintain a long-term structure stability and ensure a uniform plating/stripping behavior during long cycles. All of these characteristics synergistically contribute to the high CE, low polarization, and long life span of the MGH electrode, particularly the outstanding performance at an ultrahigh rate of $15.0 \mathrm{~mA} / \mathrm{cm}^{2}$. Therefore, reducing the local current density is an effective strategy when designing a host framework for the Li metal anode.

Acknowledgements This work was supported by the National Key Research and Development Program (Nos. 2016YFA0202500 and 2016YFA0200102), National Natural Science Foundation of China (Nos. 21676160, 21825501, 21805161, 21808121, and U1801257), and the Tsinghua University Initiative Scientific Research Program.

Open Access This article is licensed under a Creative Commons Attribution 4.0 International License, which permits use, sharing, adaptation, distribution and reproduction in any medium or format, as long as you give appropriate credit to the original author(s) and the source, provide a link to the Creative Commons licence, and indicate if changes were made. The images or other third party material in this article are included in the article's Creative Commons licence, unless indicated otherwise in a credit line to the material. If material is not included in the article's Creative Commons licence and your intended use is not permitted by statutory regulation or exceeds the permitted use, you will need to obtain permission directly from the copyright holder. To view a copy of this licence, visit http://creativecommons.org/licenses/by/4.0/.

\section{References}

1. Huang Q, Turcheniuk K, Ren XL et al (2019) Cycle stability of conversion-type iron fluoride lithium battery cathode at elevated temperatures in polymer electrolyte composites. Nat Mater 18(12):1343-1349

2. Jiang LL, Cheng XB, Peng HJ et al (2019) Carbon materials for traffic power battery. eTransportation 2:100033
3. Liang YR, Zhao CZ, Yuan $\mathrm{H}$ et al (2019) A review of rechargeable batteries for portable electronic devices. InfoMat 1(1):6-32

4. Cheng XB, Zhang R, Zhao CZ et al (2017) Toward safe lithium metal anode in rechargeable batteries: a review. Chem Rev 117(15):10403-10473

5. Shen X, Liu H, Cheng XB et al (2018) Beyond lithium ion batteries: Higher energy density battery systems based on lithium metal anodes. Energy Storage Mater 12:161-175

6. Yang Y, Davies DM, Yin YJ et al (2019) High-efficiency lithium-metal anode enabled by liquefied gas electrolytes. Joule 3(8):1986-2000

7. Song YH, Wu KJ, Zhang TW et al (2019) A nacre-inspired separator coating for impact-tolerant lithium batteries. Adv Mater 31(51):1905711

8. Xu HH, Wang SF, Manthiram A (2018) Hybrid lithium-sulfur batteries with an advanced gel cathode and stabilized lithiummetal anode. Adv Energy Mater 8(23): 1800813

9. Wang P, Qu WJ, Song WL et al (2019) Electro-chemo-mechanical issues at the interfaces in solid-state lithium metal batteries. Adv Funct Mater 29(27):1900950

10. Zhang R, Shen X, Cheng XB et al (2019) The dendrite growth in 3D structured lithium metal anodes: electron or ion transfer limitation? Energy Storage Mater 23:556-565

11. Cheng XB, Zhao CZ, Yao YX et al (2019) Recent advances in energy chemistry between solid-state electrolyte and safe lithium-metal anodes. Chem 5(1):74-96

12. Liu T, Feng XL, Jin X et al (2019) Protecting the lithium metal anode for a safe flexible lithium-air battery in ambient air. Angew Chem Int Ed 58(50):18240-18245

13. Wang SH, Yue JP, Dong W et al (2019) Tuning wettability of molten lithium via a chemical strategy for lithium metal anodes. Nat Commun 10:4930

14. Wang CZ, Wang AX, Ren LX et al (2019) Controlling Li ion flux through materials innovation for dendrite-free lithium metal anodes. Adv Funct Mater 29(49):1905940

15. Liu H, Cheng XB, Jin ZH et al (2019) Recent advances in understanding dendrite growth on alkali metal anodes. Energy Chem 1(1): 100003

16. Zhang Y, Wang CW, Pastel G et al (2018) 3D wettable framework for dendrite-free alkali metal anodes. Adv Energy Mater 8(18): 1800635

17. Zhao CZ, Duan H, Huang JQ et al (2019) Designing solid-state interfaces on lithium-metal anodes: a review. Sci China Chem 62(10):1286-1299

18. Xu R, Cheng XB, Yan C et al (2019) Artificial interphases for highly stable lithium metal anode. Matter 1(2):317-344

19. Yan C, Li HR, Chen X et al (2019) Regulating the inner Helmholtz plane for stable solid electrolyte interphase on lithium metal anodes. J Am Chem Soc 141(23):9422-9429

20. Xu R, Xiao Y, Zhang R et al (2019) Dual-phase single-ion pathway interfaces for robust lithium metal in working batteries. Adv Mater 31(19): 1970135

21. Cao X, Xu YB, Zhang LC et al (2019) Nonflammable electrolytes for lithium ion batteries enabled by ultraconformal passivation interphases. ACS Energy Lett 4(10):2529-2534

22. Shangguan XH, Xu GJ, Cui ZL et al (2019) Additive-assisted novel dual-salt electrolyte addresses wide temperature operation of lithium-metal batteries. Small 15(16):1970082

23. Zhang WD, Zhang SQ, Fan L et al (2019) Tuning the LUMO energy of an organic interphase to stabilize lithium metal batteries. ACS Energy Lett 4(3):644-650

24. Yuan YX, Wu F, Chen GH et al (2019) Porous LiF layer fabricated by a facile chemical method toward dendrite-free lithium metal anode. J Energy Chem 37:197-203

25. Gao ZG, Zhang SJ, Huang ZG et al (2019) Protection of Li metal anode by surface-coating of PVDF thin film to enhance 
the cycling performance of Li batteries. Chin Chem Lett 30(2):525-528

26. Cheng XB, Yan C, Chen X et al (2017) Implantable solid electrolyte interphase in lithium-metal batteries. Chem 2(2):258-270

27. Kong L, Jin Q, Zhang XT et al (2019) Towards full demonstration of high areal loading sulfur cathode in lithium-sulfur batteries. J Energy Chem 39:17-22

28. Zhao CZ, Chen PY, Zhang R et al (2018) An ion redistributor for dendrite-free lithium metal anodes. Sci Adv 4(11):eaat3446

29. Wu JY, Rao ZX, Cheng ZX et al (2019) Ultrathin, flexible polymer electrolyte for cost-effective fabrication of all-solid-state lithium metal batteries. Adv Energy Mater 9(46): 1902767

30. Lou JT, Wang GG, Xia Y et al (2020) Achieving efficient and stable interface between metallic lithium and garnet-type solid electrolyte through a thin indium tin oxide interlayer. J Power Sources 448:227440

31. Yan M, Liang JY, Zuo TT et al (2020) Stabilizing polymer-lithium interface in a rechargeable solid battery. Adv Funct Mater 30(6): 1908047

32. Wang G, Chen C, Chen YH et al (2020) Self-stabilized and strongly adhesive supramolecular polymer protective layer enables ultrahigh-rate and large-capacity lithium-metal anode. Angew Chem Int Ed 59(5):2055-2060

33. Huang SB, Zhang WF, Ming H et al (2019) Chemical energy release driven lithiophilic layer on $1 \mathrm{~m}^{2}$ commercial brass mesh toward highly stable lithium metal batteries. Nano Lett 19(3):1832-1837

34. Zhao H, Lei DN, He YB et al (2018) Compact 3D copper with uniform porous structure derived by electrochemical dealloying as dendrite-free lithium metal anode current collector. Adv Energy Mater 8(19): 1800266

35. Li T, Shi P, Zhang R et al (2019) Dendrite-free sandwiched ultrathin lithium metal anode with even lithium plating and stripping behavior. Nano Res 12(9):2224-2229

36. Shen X, Cheng XB, Shi P et al (2019) Lithium-matrix composite anode protected by a solid electrolyte layer for stable lithium metal batteries. J Energy Chem 37:29-34

37. Pande V, Viswanathan V (2019) Computational screening of current collectors for enabling anode-free lithium metal batteries. ACS Energy Lett 4(12):2952-2959

38. Chen X, Chen XR, Hou TZ et al (2019) Lithiophilicity chemistry of heteroatom-doped carbon to guide uniform lithium nucleation in lithium metal anodes. Sci Adv 5(2):eaau7728

39. Cao ZJ, Zhu Q, Wang S et al (2020) Perpendicular MXene arrays with periodic interspaces toward dendrite-free lithium metal anodes with high-rate capabilities. Adv Funct Mater 30(5): 1908075

40. Zhang R, Cheng XB, Zhao CZ et al (2016) Conductive nanostructured scaffolds render low local current density to inhibit lithium dendrite growth. Adv Mater 28(11):2155-2162

41. Zhai PB, Wang TS, Yang WW et al (2019) Uniform lithium deposition assisted by single-atom doping toward high-performance lithium metal anodes. Adv Energy Mater 9(18):1804019
42. Zhang XQ, Zhao CZ, Huang JQ et al (2018) Recent advances in energy chemical engineering of next-generation lithium batteries. Engineering 4(6):831-847

43. Tang C, Wang HS, Wang HF et al (2015) Spatially confined hybridization of nanometer-sized NiFe hydroxides into nitrogendoped graphene frameworks leading to superior oxygen evolution reactivity. Adv Mater 27(30):4516-4522

44. Zhang R, Chen XR, Chen X et al (2017) Lithiophilic sites in doped graphene guide uniform lithium nucleation for dendritefree lithium metal anodes. Angew Chem Int Ed 56(27):7764-7768

45. Fan YC, Wang TS, Legut D et al (2019) Theoretical investigation of lithium ions' nucleation performance on metal-doped $\mathrm{Cu}$ surfaces. J Energy Chem 39:160-169

46. Wang AX, Zhang XY, Yang YW et al (2018) Horizontal centripetal plating in the patterned voids of $\mathrm{Li} /$ graphene composites for stable lithium-metal anodes. Chem 4(9):2192-2200

47. Zhang D, Wang S, Li B et al (2019) Horizontal growth of lithium on parallelly aligned MXene layers towards dendrite-free metallic lithium anodes. Adv Mater 31(33):1901820

48. Shi P, Li T, Zhang $\mathrm{R}$ et al (2019) Lithiophilic $\mathrm{LiC}_{6}$ layers on carbon hosts enabling stable Li metal anode in working batteries. Adv Mater 31(8): 1807131

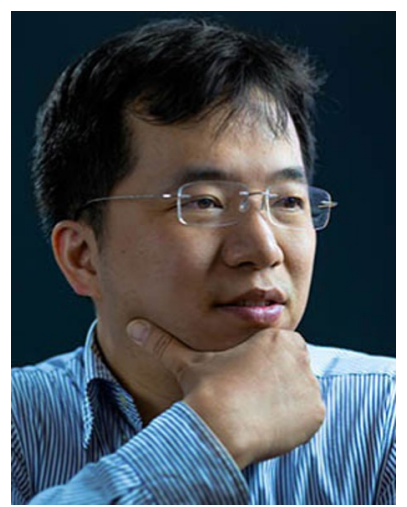

Qiang Zhang graduated from the Chemical Engineering Department, Tsinghua University, China, in 2004, where he continued doing research on mass production of carbon nanotubes and obtained his $\mathrm{PhD}$ in chemical engineering in 2009. After a short stay as a Research Associate in Case Western Reserve University, USA, in 2009, he joined the Fritz Haber Institute of the Max Planck Society, Germany, as a post-doctoral fellow. Prof. Zhang joined the faculty of Tsinghua University in 2011, and now he is a full professor of chemical engineering. He was eleted as Newton Advanced Fellowship from Royal Society, UK in 2015 and winner of the National Science Fund for Distinguished Young Scholars in 2018. Prof. Zhang is serving as an associate editor for Transactions of Tianjin University. His current research interests are advanced energy materials, including dendrite-free lithium metal anode, lithium sulfur batteries, and electrocatalysis, especially the structure design and full demonstration of advanced energy materials in working devices. 